\title{
Do We Know Who Will Drop Out? A Review of the Predictors of Dropping out of High School: Precision, Sensitivity and Specificity ${ }^{1}$ Alex J. Bowers ${ }^{2}$, Ryan Sprott ${ }^{3}$ \& Sherry Taff $^{3}$
}

\begin{abstract}
The purpose of this study is to review the literature on the most accurate indicators of students at risk of dropping out of high school. We used Relative Operating Characteristic (ROC) analysis to compare the sensitivity and specificity of 110 dropout flags across 36 studies. Our results indicate that 1) ROC analysis provides a means to compare the accuracy of different dropout indicators, 2) the majority of dropout flags to date have high precision yet lack accuracy, 3) longitudinal growth models provided the most accurate flags, while 4) the most accurate crosssectional flags examine low or failing grades. We provide recommendations for future policy and practice.
\end{abstract}

Keywords: Dropout, dropout characteristics, dropout identification, dropout prediction, dropout research, ROC, relative operating characteristic, receiver operating characteristic, growth mixture models, grades.

\section{INTRODUCTION}

For students who fail to graduate from high school, a long history of research has demonstrated that on average, in comparison to graduates, these students experience higher rates of unemployment and incarceration and lower overall lifetime earnings and life expectancy (Berktold \& Carroll, 1998; Jemal, Ward, Anderson, Murray, \& Thun, 2008; Moretti, 2007; Muenning, 2007; Rouse, 2007; Swanson, 2009; Waldfogel, Garfinkel, \& Kelly, 2007). In the United States, graduation rates are estimated to average between $70 \%$ and $80 \%$ nationally (Balfanz, Bridgeland, Moore, \& Hornig Fox, 2010; Cataldi, Laird, \& KewalRamani, 2009; Kaufman, 2004). However, for some schools, specifically schools in urban and poor contexts, graduation rates have been shown to be as low as $50 \%$ or less (Balfanz, et al., 2010; Balfanz \& Legters, 2006; Swanson, 2004). Since the 1970s, many demographic factors have been associated with dropping out of school (Rumberger, 1987, 2004) including increased rates of drop out among males, African Americans, Hispanics, low socioeconomic (SES) families, as well as schools in urban and rural contexts. However, beyond demographic variables, the central focus of the current study is to ask: What do we know about how well school malleable factors predict if a student will drop out or graduate?

\footnotetext{
1 This document is a pre-print of this manuscript, published in 2013 in The High School Journal. Recommended Citation: Bowers, A.J., Sprott, R., Taff, S.A. (2013) Do we Know Who Will Drop Out? A Review of the Predictors of Dropping out of High School: Precision, Sensitivity and Specificity. The High School Journal. 96(2), 77-100. doi:10.1353/hsj.2013.0000

${ }^{2}$ Teachers College, Columbia University; Bowers@tc.edu; 525 W. $120^{\text {th }}$ Street, New York, New York 10027.

ORCID: 0000-0002-5140-6428, ResearcherID: C-1557-2013

${ }^{3}$ The University of Texas at San Antonio.

Note: This document last updated July 30, 2013.
}

Bowers, Sprott \& Taff (2013) Do We Know Who Will Dropout?
This issue is important not only in helping understand who will drop out, but also aids in a school's decision to provide dropout interventions to students deemed at-risk (Gleason \& Dynarski, 2002). If a dropout predictor is not accurate, then some students will be misidentified as likely to drop out when they would have graduated without intervention. As noted by Gleason and Dynarski (2002), this issue leads to inefficient management of limited school district resources, as schools are potentially funding expensive dropout prevention initiatives to students who do not require intervention. In addition, students misidentified as at risk of dropping out, when in fact they would not have dropped out, could conceivably be categorized under a type of at-risk deficit model, negatively impacting their school achievement as they may be pulled out of the regular curriculum for dropout interventions or experience other adverse consequences of the misapplied at-risk label. The reverse situation is also a major problem: If a predictor is inaccurate, then many students who do eventually drop out are never identified as at-risk, and thus the school district is unaware of the issue and does not provide an intervention to students who may need it. Indeed, some studies have estimated that many dropout flags only accurately identify about $50 \%$ to $60 \%$ of the students who do eventually drop out (Balfanz, Herzog, \& MacIver, 2007; Gleason \& Dynarski, 2002; Janosz, Archambault, Morizot, \& Pagani, 2008). This means that large percentages of students who are identified by dropout flags do not drop out, and conversely a large percentage of students who do drop out of high schools are not identified by their school districts.

Nevertheless, there is a perception in policy and the research literature that we know who will drop out. As stated by Troob (1985), "[Their study] supports the perception that most future dropouts can be identified at the beginning of their high school careers" (p. 1). But is this perception true? Claims across the dropout prediction literature are extremely varied, with many reporting specific problems with dropout flags, to others that state that they are able to predict early on who will drop out, to still other studies that state that their flags are almost $100 \%$ predictive. However, the literature is plagued by inconsistent language, and to date, no study has attempted to compare each of the reported dropout flags across the studies on a standard metric, comparing the claims of each study on precision, sensitivity and specificity. This inconsistency has created a hodgepodge of claims as to the accuracy of the tested dropout flags across the literature that is difficult to evaluate in an effort to help schools, districts, researchers and policymakers find and employ accurate indicators of student dropout risk.

\section{Purpose}

The purpose of this study is threefold. First, we aim to comprehensively examine the dropout prediction literature over the past 30 years and present a synthesis of each of the calculations from across the studies by recalculating and reporting the 
precision, sensitivity and specificity from each study, whether it was originally reported or not. Second, we outline a method from the signal detection theory literature for comparing each dropout flag for precision, sensitivity and specificity so that each dropout indicator can be compared as to its accuracy, known as a Relative Operating Characteristic (ROC). Third, we present a comparison of each of the dropout flags using a ROC analysis to identify which predictors of high school dropout are the most accurate and usable by schools, districts, policymakers and researchers.

\section{METHODS}

\section{Study Selection and Inclusion Criteria}

As a review of the literature on the most accurate dropout flags that correctly identify students who drop out and do not misidentify students who graduate as at risk of dropping out, we aimed to include studies from the past 30 years that presented empirical results on dropout predictors. Our criteria list for eligibility for each study was: (a) The study focused on high school dropout prediction; (b) The study examined school-wide characteristics, all students in the school were included in the study, and the study was not specific to one subgroup of students (such as students with a learning disability); (c) The study focused on student-level, not school-level, dropout characteristics; (d) The study contained quantitative data that fit our specific requirements for recalculating precision, sensitivity and specificity, such that a cross-tabulation contingency event table could be constructed for each reported dropout flag.

To create an encompassing search of high school dropout prediction literature, we performed Boolean searches in JSTOR, ERIC, Educational Full Text Wilson Web and Google Scholar. We searched literature published after 1979. We used various search strings to explore the breadth of the articles pertaining to high school dropout prediction. The Boolean phrase "(dropout*) AND (Indicat* OR Identif* OR Predict*) AND (School* or edu*)" serves as an example of one of the more effective search phrases. This specific search yielded 843 articles in EBSCO, 1437 in HW Full Text, 15322 in JSTOR, and 14400 in Google Scholar (not mutually exclusive). In addition we included Worldcat and Google Books to search for books relating to our study. Throughout the database research process, reference sections of applicable articles, foundational pieces and dissertations were mined for further resources that may have been missed through the Boolean searches. This resulted in 6,434 studies. After reading the article titles and abstracts yielded by the initial searches, we decided to remove dissertations as well as publications that only studied a specific school subpopulation to add the appropriate specificity to our paper (i.e., gender, ethnicity, socioeconomic levels, learning disabilities). We omitted studies that analyzed school effects on students' likelihood to drop out, such as how school size impacted blah

GED/alternative education certificate, as indicators of dropping out. For the studies that included transfer (Balfanz, et al., 2007; Mensch \& Kandel, 1988), the authors argued that the final student outcomes of students who transferred out of the studied districts could not be determined. For the GED/alternative education inclusion, studies that included this outcome took the perspective of identifying indicators of on-time high school graduation with a regular high school diploma, given the literature on the nonequivalence of the GED in comparison to a regular high school diploma (Cameron \& Heckman, 1993; Tyler, 2003).

Table 1: Dropout study locations, graduation years and definitions student dropout rates. Thus, the present study includes studies that predicted dropout likelihood based on student-level characteristics using school-wide samples. This resulted in 301 studies that were read in full.

The final stage narrowed down these 301 further to 140 studies after parceling out those that initially seemed usable but upon reading the full text did not meet the inclusion criteria by either not having a school-wide sample or by not reporting quantifiable data. Finally, these 140 articles were examined more closely to determine whether the articles included the data we required. Thirty-six of these articles contained the necessary data for the new calculations discussed below. We derived from the reporting in each of these 36 articles the following information: the number of students in the sample with the dropout indicator, the number of the students without the dropout indicator, the number of students with the flag who dropped out, and the number of students without the flag who dropped out. We used this information to determine the precision, specificity, and sensitivity of each study's dropout predictor. Multiple articles reported multiple dropout indicators, hence from the 36 articles, we report on 110 dropout flags.

\section{Sample Characteristics and Dropout Definitions}

Table 1 provides sample characteristics of each of the 36 studies, including the database and sample location analyzed in each study, the projected graduation year of the cohort studied, and an indication of how each study defined a dropout. If a study used a nationally representative sample, then the location is denoted as U.S. As is evident from Table 1, many of the studies are nationallevel studies, examining samples collected by NCES, such as High School and Beyond (HS\&B), the National Education Longitudinal Study of 1988 (NELS:88), or the Education Longitudinal Study of 2002 (ELS:2002). In addition, large school districts, such as Chicago Public Schools and Los Angeles Unified School District, as well as many smaller school districts are represented as both named and unnamed across multiple studies from the U.S. and Canada. Also, a broad range of projected graduation years is represented across the studies,

from 1975 through 2006.

While the definition of who is a "dropout" on the surface appears to be simple, such as all students who did not receive a high school diploma, how a dropout is defined is an issue across the literature. High school students have a broad range of options in the timing of completing their degrees (Bowers, 2010b; Cameron \& Heckman, 1993; Kronick \& Hargis, 1998; Pallas, 1989, 1993, 2003), such that students may drop out and then return to school to complete their degree at a later date, can transfer to other schools, or can complete a GED or alternative education degree. As presented in Table 1, multiple studies noted in the text of their methods that they included these other outcomes, such as transfer or a

\section{Comparative Analysis Method}

To compare the accuracy of each of the 110 dropout indicators reported across the 36 studies, we first recorded the reported numbers from each manuscript. Of note, none of the studies correctly reported all three calculations of precision, sensitivity and specificity. Many of the studies reported the overall sample size, and then variations on the percentages of dropout students with the dropout indicator, or students with the indicator who dropped out. In many instances these percentages were reported as whole 


\begin{tabular}{|c|c|c|c|c|c|c|}
\hline Study & Database & Location & $\begin{array}{l}\text { Projected } \\
\text { Graduatio } \\
\text { n Year }\end{array}$ & $\begin{array}{c}\text { Included in } \\
\text { No High } \\
\text { School } \\
\text { Diploma }\end{array}$ & $\frac{\text { Dropout D }}{\text { Transfer }}$ & $\begin{array}{l}\text { Definition } \\
\text { GED/ } \\
\text { Alt Ed }\end{array}$ \\
\hline \multicolumn{2}{|c|}{ Allensworth \& Easton (2007) Chicago Public Schools } & Chicago & 2005 & $\mathrm{X}$ & & \\
\hline Austin ISD (1982) & Austin Independent School District & Austin & 1983 & $x$ & & \\
\hline Balfanz et al. (2007) & School district of Philadelphia & Philadelphia & 2004 & $x$ & $x$ & \\
\hline Bowers (2010a) & District transcript records & Mid-West district & 2006 & $x$ & & $x$ \\
\hline Bowers (2010b) & District transcript records & Mid-West district & 2006 & $x$ & & $x$ \\
\hline Bowers \& Sprott (2012a) & $\begin{array}{l}\text { Education Longitudinal Study of } 2002 \\
\text { (ELS:2002) }\end{array}$ & U.S. & 2004 & $x$ & & $x$ \\
\hline Curtis et al. (1983) & Austin Independent School District & Austin & 1983 & $\mathrm{x}$ & & \\
\hline Dalton et al. (2009) & $\begin{array}{l}\text { Education Longitudinal Study of } 2002 \\
\text { (ELS:2002) }\end{array}$ & U.S. & 2004 & $x$ & & \\
\hline Doss (1986) & Austin Independent School District & Austin & 1983 & $x$ & & \\
\hline Duchesne et al. (2008) & Quebec Ministry of Education & Quebec & 2000 & $x$ & & \\
\hline Eide \& Showalter (2001) & $\begin{array}{l}\text { High School and Beyond (HS\&B): } \\
1980 \text { Sophomore }\end{array}$ & U.S. & 1982 & $x$ & & \\
\hline Ekstrom et al. (1986) & $\begin{array}{l}\text { High School and Beyond (HS\&B): } \\
1980 \text { Sophomore }\end{array}$ & U.S. & 1982 & $\mathrm{x}$ & & \\
\hline $\begin{array}{l}\text { Ensminger \& Slusarcick } \\
\text { (1992) }\end{array}$ & Chicago Public Schools & Chicago & 1978 & $\mathrm{x}$ & & \\
\hline Finn et al. (2008) & $\begin{array}{l}\text { National Education Longitudinal Study } 1988 \\
\text { (NELS:88) }\end{array}$ & U.S. & 1992 & $\mathrm{x}$ & & \\
\hline Frazer (1991) & Austin Independent School District & Austin & 1991-1996 & $x$ & & \\
\hline French \& Conrad (2001) & Pacific Northwest & $\begin{array}{l}\text { Pacific Northwest } \\
\text { District }\end{array}$ & $\begin{array}{l}\text { No year } \\
\text { given }\end{array}$ & $x$ & & \\
\hline Gleason \& Dynarski (2002) & $\begin{array}{l}\text { School Dropout Demonstration Assistance } \\
\text { Program }\end{array}$ & $\begin{array}{l}\text { Dallas TX, Phoenix } \\
\text { AZ, Grand Rapids } \\
\text { MI, Santa Ana CA }\end{array}$ & 1995-1999 & $x$ & & \\
\hline Hess \& Lauber (1985) & Chicago Public Schools & Chicago & $1982-1984$ & $x$ & & \\
\hline HRSD (2006) & School Leavers Survey & Canada & 1993-1995 & $x$ & & \\
\hline Janosz et al. (2008) & $\begin{array}{l}\text { New Solutions longitudinal data set (2002- } \\
\text { 2005) }\end{array}$ & Quebec & 2005 & $x$ & & \\
\hline Kupersmidt \& Coie (1990) & Coie \& Associates Longitudinal Study & $\begin{array}{l}\text { Durham County, } \\
\text { North Carolina }\end{array}$ & 1983 & $x$ & & \\
\hline Lee \& Staff (2007) & $\begin{array}{l}\text { National Education Longitudinal Study } 1988 \\
\text { (NELS:88) }\end{array}$ & U.S. & 1992 & $\mathrm{x}$ & & \\
\hline Mahoney (2000) & Carolina Longitudinal Study (CLS) & $\begin{array}{l}\text { Southeastern } \\
\text { United States }\end{array}$ & 1986, 1989 & $x$ & & $x$ \\
\hline McCaul (1989) & $\begin{array}{l}\text { High School and Beyond (HS\&B): } \\
1980 \text { Sophomore and } 1982 \text { follow-up surveys }\end{array}$ & U.S. & 1982 & $x$ & & \\
\hline McNeal (1997) & $\begin{array}{l}\text { High School and Beyond (HS\&B): } \\
1980 \text { Sophomore and } 1982 \text { follow-up surveys }\end{array}$ & U.S. & 1982 & $\mathrm{x}$ & & \\
\hline Mensch \& Kandel (1988) & $\begin{array}{l}\text { National Longitudinal Survey of Young Adults } \\
\text { (aged 19-27 in 1984) }\end{array}$ & U.S. & $1975-1983$ & $\mathrm{x}$ & $X$ & $\mathrm{X}$ \\
\hline Muthén (2004) & $\begin{array}{l}\text { Longitudinal Study of Youth (LSAY) from cohort } \\
2 \text {, from Grade } 7 \text { through } 12 \text { in } 1987\end{array}$ & tU.S. & 1992 & $x$ & & \\
\hline Pagani et al. (2008) & $\begin{array}{l}\text { Quebec Longitudinal Study of Kindergarten } \\
\text { Children (QLSKC) spring of } 1986 \text { and } 1987\end{array}$ & Quebec & 1999, 2000 & $x$ & & \\
\hline Roderick (1994) & Fall River school district transcript records & Fall River, Ma. & 1996 & $x$ & & \\
\hline Sandefur et al. (1992) & $\begin{array}{l}\text { National Longitudinal Survey of Young Adults } \\
\text { waves } 1979-1985 \text {, study confined to } 14-17 \text { in } \\
1979\end{array}$ & U.S. & 1985 & $\mathrm{x}$ & & \\
\hline
\end{tabular}

Table 1 continued

Bowers, Sprott \& Taff (2013) Do We Know Who Will Dropout? 


\begin{tabular}{|c|c|c|c|c|c|c|}
\hline \multirow[b]{2}{*}{ Study } & \multirow[b]{2}{*}{ Database } & \multirow[b]{2}{*}{ Location } & \multirow[b]{2}{*}{$\begin{array}{c}\text { Projected } \\
\text { Graduation } \\
\text { Year }\end{array}$} & \multicolumn{3}{|c|}{ Included in Dropout Definition } \\
\hline & & & & $\begin{array}{l}\text { No High } \\
\text { School } \\
\text { Diploma }\end{array}$ & Transfer & $\begin{array}{l}\text { GED/ } \\
\text { Alt Ed }\end{array}$ \\
\hline Silver et al. (2008) & Los Angeles Unified School District & Los Angeles & 2005 & $x$ & & \\
\hline South et al. (2007) & $\begin{array}{l}\text { National Longitudinal Study of Adolescent } \\
\text { Health }\end{array}$ & U.S. & $1995-2000$ & $x$ & & $x$ \\
\hline Suh and Suh (2007) & $\begin{array}{l}\text { National Longitudinal Survey of Young Adults } \\
\text { (1997) }\end{array}$ & U.S. & 2000 & $x$ & & \\
\hline Troob (1985) & $\begin{array}{l}\text { New York City Board of Education: Student } \\
\text { Automated Record-Keeping System (SARK) }\end{array}$ & New York & 1983, 1984 & $x$ & & \\
\hline Warren and Lee (2003) & $\begin{array}{l}\text { National Education Longitudinal Study } 1988 \\
\text { (NELS:88) }\end{array}$ & U.S. & 1992 & $\mathrm{X}$ & & \\
\hline
\end{tabular}

numbers, or only to one decimal place, thus we back-calculated from the percentages to get the overall numbers of students in each group, rounding up to whole numbers when required. In addition, some studies reported the percentages of students who graduated with the flags, rather than dropped out; in these instances we recalculated the reported numbers as dropout indicators, such as taking one minus the reported graduation flag percentage to get the dropout percentage. Furthermore, many studies first report the overall number of students with the dropout indicators and then go on to conduct inferential statistics, such as logistic regression, failing to report the posterior probabilities of the statistics, reporting only regression coefficients. In these cases, we included the study data from the descriptives only, since it was not possible to deduce the required frequency information without the posterior probabilities. Thus, unless otherwise noted, calculations for each study's dropout flags are based on descriptive cross-tabulations.

The purpose of the present study is to highlight, encourage, and provide an example of the usefulness of providing accuracy measures across the dropout indicator research and to help move the field towards a more consistent reporting structure. As will be detailed further below, an analysis across the literature that would take the form of a meta-analysis is outside the scope of the present study due to this current lack of consistent reporting across the studies. Thus, we turned to signal detection theory to examine the accuracy of the identified dropout flags.

Following the recommendations of the signal detection and diagnostics systems accuracy theory literature (Hanley \& McNeil, 1982; Swets, 1988; Vivo \& Franco, 2008; Zwieg \& Campbell, 1993), our analysis of each study included calculations for precision, sensitivity, and specificity (see Figure 1). Figure 1 outlines a contingency table (crosstabs) in which the event under consideration is if a student drops out or graduates (columns). A dropout indicator predicts if the student will drop out or graduate (rows). This type of event table mirrors issues with Type I and Type II errors (Rogosa, 2005), in that true-positives and truenegatives are correct predictions of dropouts and graduates, but false-positives are Type I errors that reflect students with a dropout flag who graduate, while false-negatives are Type II errors that reflect students predicted to graduate who then drop out. We are interested here in examining the interplay between these different prediction outcomes in dropout indicator studies. Thus our analysis included calculations for precision, which is the true-positives Table 2: Dropout Indicator Results by Study

Bowers, Sprott \& Taff (2013) Do We Know Who Will Dropout? divided by the total number of students with the flag, the truepositive proportion (sensitivity) which is the true-positives divided by the total number of actual dropouts, the true-negative proportion (specificity) which is the true-negatives divided by the total number of graduates, and the false-positive proportion (1specificity) which is the false-positives divided by the total number of graduates. None of the studies reviewed for this analysis reported all four of these indicators of accuracy, with almost all studies reporting precision and many reporting sensitivity, but almost none reporting specificity or the false-positive proportion. In the parlance of signal detection theory (Swets, 1988), the two most important calculations for considering the accuracy of a predictor are the true-positive proportion and the false-positive proportion, known as "hits" versus "false alarms." Unless a detection system is perfect, there will always be a trade-off between hits and false alarms, in that as one attempts to maximize the number of hits by casting a wider net, one must also be conscious of the number of false alarms that the wider net may also end up catching mistakenly. It is exactly this issue that is the focus of the present study, which has to date rarely been addressed in the dropout indicator literature.

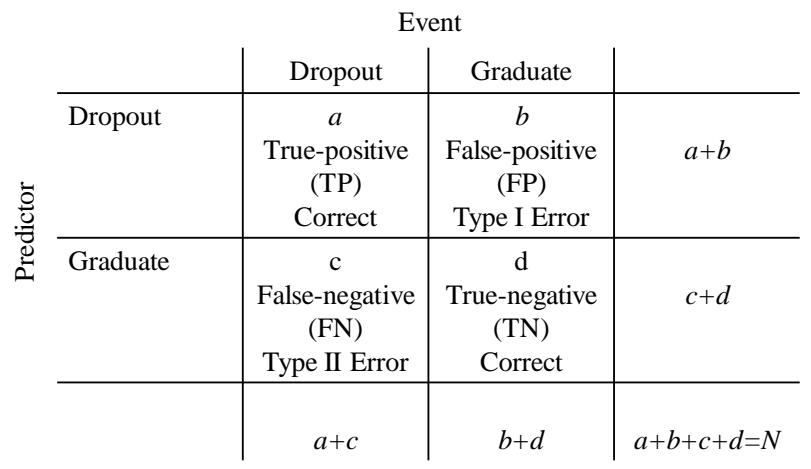

$\begin{array}{lll}\text { Precision } & =\mathrm{a} /(\mathrm{a}+\mathrm{b}) & \text { Positive Predictive Value } \\ \text { True-Positive Proportion } & =\mathrm{a} /(\mathrm{a}+\mathrm{c}) & \text { Sensitivity } \\ \text { True-Negative Proportion } & =\mathrm{d} /(\mathrm{b}+\mathrm{d}) & \text { Specificity } \\ \text { False-Positive Proportion } & =\mathrm{b} /(\mathrm{b}+\mathrm{d}) & \text { 1-Specificity }\end{array}$

Figure 1: Event table for calculating dropout contingency proportions 


\begin{tabular}{|c|c|c|c|c|c|c|c|c|c|}
\hline ID & Study & $\begin{array}{l}\text { Grade } \\
\text { Level }\end{array}$ & Dropout Indicator & $\begin{array}{l}\text { Sample } \\
\text { Size }\end{array}$ & $\begin{array}{l}\text { Dropout } \\
\text { Rate }\end{array}$ & $\begin{array}{l}\text { Precision } \\
\text { Positive } \\
\text { Predictive } \\
\text { Value }\end{array}$ & $\begin{array}{c}\text { Sensitivity } \\
\text { True- Positive } \\
\text { Proportion }\end{array}$ & $\begin{array}{c}\text { Specificity } \\
\text { True- Negative } \\
\text { Proportion }\end{array}$ & $\begin{array}{c}\text { False- } \\
\text { Positive } \\
\text { Proportion } \\
\text { (1-Specificity) }\end{array}$ \\
\hline 1 & $\begin{array}{l}\text { Allensworth \& } \\
\text { Easton (2007) }\end{array}$ & 9 & $\begin{array}{l}\text { On Track indicator, low course credits and more } \\
\text { than one failed course }\end{array}$ & 20803 & 0.426 & 0.780 & 0.751 & 0.843 & 0.157 \\
\hline 2 & & $9-12$ & 3 or more semester F's & 20340 & 0.411 & 0.806 & 0.648 & 0.891 & 0.109 \\
\hline 3 & Austin ISD (1982) & 9 & Retention, student in grade 8 or lower & 3899 & 0.242 & 0.487 & 0.453 & 0.848 & 0.152 \\
\hline 4 & Balfanz et al. (2007) & 6 & Attended less than or equal to $80 \%$ of the time & 12037 & 0.572 & 0.830 & 0.233 & 0.936 & 0.064 \\
\hline 5 & & 6 & Failed Math & 12037 & 0.572 & 0.810 & 0.212 & 0.934 & 0.066 \\
\hline 6 & & 6 & Failed English & 12037 & 0.572 & 0.820 & 0.168 & 0.951 & 0.049 \\
\hline 7 & & 6 & Suspended out of school & 12037 & 0.572 & 0.800 & 0.098 & 0.967 & 0.033 \\
\hline 8 & & 6 & Unsatisfactory behavior & 12037 & 0.572 & 0.710 & 0.505 & 0.725 & 0.275 \\
\hline 9 & & 6 & $\begin{array}{l}\text { One or more flags (low attendance, unsatisfactory } \\
\text { behavior, fail math or English) }\end{array}$ & 12037 & 0.572 & 0.710 & 0.595 & 0.675 & 0.325 \\
\hline 10 & & 6 & Any one flag & 12037 & 0.572 & 0.636 & 0.323 & 0.753 & 0.247 \\
\hline 11 & & 6 & Any two flags & 12037 & 0.572 & 0.791 & 0.153 & 0.946 & 0.054 \\
\hline 12 & & 6 & Any three flags & 12037 & 0.572 & 0.863 & 0.078 & 0.983 & 0.017 \\
\hline 13 & & 6 & All four flags & 12037 & 0.572 & 0.923 & 0.044 & 0.995 & 0.005 \\
\hline 14 & Bowers (2010b) & $7-12$ & $\begin{array}{l}\text { Retention, student ever retained in any grade } \\
\text { level }\end{array}$ & 193 & 0.244 & 0.909 & 0.426 & 0.986 & 0.014 \\
\hline 15 & & $7-12$ & Low non-cumulative GPA (D or lower) & 193 & 0.244 & 0.514 & 0.809 & 0.753 & 0.247 \\
\hline 16 & Bowers (2010a) & $\mathrm{K}-6$ & $\begin{array}{l}\text { Hierarchical cluster analysis of non-cumulative } \\
\text { course grades, K-6 }\end{array}$ & 145 & 0.186 & 0.258 & 0.630 & 0.585 & 0.415 \\
\hline 17 & & $\mathrm{~K}-8$ & $\begin{array}{l}\text { Hierarchical cluster analysis of non-cumulative } \\
\text { course grades, K-8 }\end{array}$ & 154 & 0.214 & 0.356 & 0.939 & 0.537 & 0.463 \\
\hline 18 & & $\mathrm{~K}-12$ & $\begin{array}{l}\text { Hierarchical cluster analysis of non-cumulative } \\
\text { course grades, K-12 }\end{array}$ & 186 & 0.237 & 0.379 & 0.886 & 0.549 & 0.451 \\
\hline 19 & $\begin{array}{l}\text { Bowers \& Sprott } \\
\text { (2012a) }\end{array}$ & $9-12$ & $\begin{array}{l}\text { Growth mixture modeling using non-cumulative } \\
\text { semester GPA }\end{array}$ & 5400 & 0.090 & 0.336 & 0.918 & 0.821 & 0.180 \\
\hline 20 & $\begin{array}{l}\text { Croninger and Lee } \\
\text { (2001) }\end{array}$ & $10-12$ & $\begin{array}{l}\text { 3-5 Social Factors (low SES, ESL, non-white, } \\
\text { single parent, mother dropped out) }\end{array}$ & 10979 & 0.114 & 0.106 & 0.405 & 0.561 & 0.439 \\
\hline 21 & & $10-12$ & $\begin{array}{l}\text { Academic Risk (GPA }<2.0 \text {, retained, will not go to } \\
\text { college, high discipline) }\end{array}$ & 10979 & 0.114 & 0.237 & 0.708 & 0.707 & 0.293 \\
\hline 22 & Curtis et al. (1983) & $9-12$ & D grade average & 3907 & 0.241 & 0.810 & 0.199 & 0.985 & 0.015 \\
\hline 23 & & $9-12$ & Retention, student in grade 8 or lower & 3907 & 0.241 & 0.486 & 0.453 & 0.848 & 0.152 \\
\hline 24 & Dalton et al. (2009) & $10-12$ & Retention, age 17 or older in grade 10 & 15360 & 0.066 & 0.276 & 0.284 & 0.947 & 0.053 \\
\hline 25 & & $10-12$ & Non-native English Speaker & 15360 & 0.066 & 0.103 & 0.208 & 0.872 & 0.128 \\
\hline 26 & & $10-12$ & Lowest SES quartile & 15360 & 0.066 & 0.124 & 0.457 & 0.772 & 0.228 \\
\hline 27 & & $10-12$ & Parent's education HS or less & 15360 & 0.066 & 0.112 & 0.453 & 0.746 & 0.254 \\
\hline 28 & & $10-12$ & Changed schools 3 or more times & 15360 & 0.066 & 0.083 & 0.245 & 0.808 & 0.192 \\
\hline 29 & & $10-12$ & Student does not expect to graduate HS & 15360 & 0.066 & 0.299 & 0.045 & 0.992 & 0.008 \\
\hline 30 & & $10-12$ & $\begin{array}{l}\text { English teacher does not expect student to } \\
\text { graduate from HS }\end{array}$ & 15360 & 0.066 & 0.342 & 0.150 & 0.980 & 0.020 \\
\hline
\end{tabular}


Table 2 continued

\begin{tabular}{|c|c|c|c|c|c|c|c|c|c|}
\hline ID & Study & $\begin{array}{l}\text { Grade } \\
\text { Level }\end{array}$ & Dropout Indicator & $\begin{array}{l}\text { Sample } \\
\text { Size }\end{array}$ & $\begin{array}{l}\text { Dropout } \\
\text { Rate }\end{array}$ & $\begin{array}{l}\text { Precision } \\
\text { Positive } \\
\text { Predictive } \\
\text { Value }\end{array}$ & $\begin{array}{c}\text { Sensitivity } \\
\text { True- Positive } \\
\text { Proportion }\end{array}$ & $\begin{array}{l}\text { Specificity } \\
\text { True- } \\
\text { Negative } \\
\text { Proportion }\end{array}$ & $\begin{array}{c}\text { False- } \\
\text { Positive } \\
\text { Proportion } \\
\text { (1-Specificity) }\end{array}$ \\
\hline 31 & & $10-12$ & $\begin{array}{l}\text { Teachers rate student as not relating well to } \\
\text { others }\end{array}$ & 15360 & 0.066 & 0.179 & 0.116 & 0.962 & 0.038 \\
\hline 32 & & $10-12$ & Teachers rate student as disruptive in class & 15360 & 0.066 & 0.137 & 0.143 & 0.936 & 0.064 \\
\hline 33 & & $10-12$ & Zero hours of homework completed per week & 15360 & 0.066 & 0.123 & 0.136 & 0.931 & 0.069 \\
\hline 34 & & $10-12$ & $\begin{array}{l}\text { Lowest standardized test quartile math and } \\
\text { reading }\end{array}$ & 15360 & 0.066 & 0.133 & 0.475 & 0.781 & 0.219 \\
\hline 35 & & $10-12$ & $\begin{array}{l}\text { Less than } 10 \text { credits by spring of sophomore } \\
\text { year }\end{array}$ & 15360 & 0.066 & 0.546 & 0.290 & 0.983 & 0.017 \\
\hline 36 & Doss $(1986)^{*}$ & 9 & $\begin{array}{l}\text { Four Flags (low GPA, retained, gender, } \\
\text { ethnicity, discipline) }\end{array}$ & 3038 & 0.214 & 0.483 & 0.698 & 0.797 & 0.203 \\
\hline 37 & $\begin{array}{l}\text { Duchesne et al. } \\
(2008)\end{array}$ & K-6 & Anxiety Trajectories & 1817 & 0.308 & 0.361 & 0.597 & 0.531 & 0.469 \\
\hline 38 & $\begin{array}{l}\text { Eide and Showalter } \\
\text { (2001) }\end{array}$ & $10-12$ & Retention, student ever repeated a grade & 7809 & 0.165 & 0.367 & 0.312 & 0.894 & 0.106 \\
\hline 39 & $\begin{array}{l}\text { Ekstrom et al. } \\
\text { (1986) }\end{array}$ & $10-12$ & Cut classes & 24000 & 0.150 & 0.276 & 0.540 & 0.750 & 0.250 \\
\hline 40 & & $10-12$ & Had disciplinary problems & 24000 & 0.150 & 0.311 & 0.410 & 0.840 & 0.160 \\
\hline 41 & & $10-12$ & Suspended or put on probation & 24000 & 0.150 & 0.354 & 0.310 & 0.900 & 0.100 \\
\hline 42 & & $10-12$ & Serious trouble with the law & 24000 & 0.150 & 0.328 & 0.130 & 0.953 & 0.047 \\
\hline 43 & & $10-12$ & Not interested in school & 24000 & 0.150 & 0.252 & 0.400 & 0.790 & 0.210 \\
\hline 44 & & $10-12$ & Not satisfied with the way education is going & 24000 & 0.150 & 0.238 & 0.550 & 0.690 & 0.310 \\
\hline 45 & & $10-12$ & Does not like to work hard in school & 24000 & 0.150 & 0.194 & 0.600 & 0.560 & 0.440 \\
\hline 46 & & $10-12$ & Close friend does not attend class regularly & 24000 & 0.150 & 0.312 & 0.180 & 0.930 & 0.070 \\
\hline 47 & & $10-12$ & Close friend is not popular & 24000 & 0.150 & 0.218 & 0.190 & 0.880 & 0.120 \\
\hline 48 & & $10-12$ & Close friend does not get good grades & 24000 & 0.150 & 0.219 & 0.270 & 0.830 & 0.170 \\
\hline 49 & & $10-12$ & Close friend is not interested in school & 24000 & 0.150 & 0.218 & 0.490 & 0.690 & 0.310 \\
\hline 50 & & $10-12$ & Close friend does not plan on going to college & 24000 & 0.150 & 0.230 & 0.560 & 0.670 & 0.330 \\
\hline 51 & $\begin{array}{l}\text { Ensminger and } \\
\text { Slusarcick (1992) }\end{array}$ & 1 & Low Grades in Grade 1, C or less & 864 & 0.508 & 0.599 & 0.620 & 0.572 & 0.428 \\
\hline 52 & Finn et al. (2008) & $8-10$ & $\begin{array}{l}\text { High misbehavior, four or more different } \\
\text { misbehavior flags }\end{array}$ & 16489 & 0.105 & 0.336 & 0.231 & 0.947 & 0.053 \\
\hline 53 & Frazer (1991) & $9-12$ & $\begin{array}{l}\text { Texas At-Risk Category (retained, low math } \\
\text { and reading skills, low or failing grades) }\end{array}$ & 16657 & 0.187 & 0.272 & 0.665 & 0.590 & 0.410 \\
\hline 54 & $\begin{array}{l}\text { French and Conrad } \\
\text { (2001) }\end{array}$ & 8 & $\begin{array}{l}\text { Grade } 8 \text { peer rejection and antisocial } \\
\text { categories }\end{array}$ & 218 & 0.133 & 0.217 & 0.448 & 0.751 & 0.249 \\
\hline 55 & & 8 & $\begin{array}{l}\text { Grade } 10 \text { peer rejection and antisocial } \\
\text { categories }\end{array}$ & 610 & 0.062 & 0.095 & 0.421 & 0.734 & 0.266 \\
\hline 56 & $\begin{array}{l}\text { Gleason and } \\
\text { Dynaraski (2002) }\end{array}$ & $10-12$ & HS Multiple Regression using multiple flags + & 2615 & 0.146 & 0.421 & 0.432 & 0.898 & 0.102 \\
\hline 57 & $\begin{array}{l}\text { Hess and Lauber } \\
\text { (1985) }\end{array}$ & 9 & Retention, student age 16 or older in grade 9 & 29942 & 0.428 & 0.621 & 0.048 & 0.978 & 0.022 \\
\hline 58 & & 8 & Low reading scores & 29942 & 0.428 & 0.549 & 0.534 & 0.673 & 0.327 \\
\hline 59 & HRSD (2006) & $9-12$ & Student lives in single parent household & 9460 & 0.162 & 0.270 & 0.250 & 0.869 & 0.131 \\
\hline 60 & & $9-12$ & $\begin{array}{l}\text { Student did not live with either parent in the } \\
\text { last school year }\end{array}$ & 9460 & 0.162 & 0.301 & 0.130 & 0.942 & 0.058 \\
\hline 61 & & $9-12$ & $\begin{array}{l}\text { Student reports that parents do not consider } \\
\text { HS completion very important }\end{array}$ & 9460 & 0.162 & 0.489 & 0.211 & 0.957 & 0.043 \\
\hline 62 & & $9-12$ & High risk group ++ & 9460 & 0.162 & 0.270 & 0.667 & 0.652 & 0.348 \\
\hline
\end{tabular}


Table 2 continued

\begin{tabular}{|c|c|c|c|c|c|c|c|c|c|}
\hline ID & Study & $\begin{array}{l}\text { Grade } \\
\text { Level }\end{array}$ & Dropout Indicator & $\begin{array}{l}\text { Sample } \\
\text { Size }\end{array}$ & $\begin{array}{l}\text { Dropout } \\
\text { Rate }\end{array}$ & $\begin{array}{l}\text { Precision } \\
\text { Positive } \\
\text { Predictive } \\
\text { Value }\end{array}$ & $\begin{array}{c}\text { Sensitivity } \\
\text { True- Positive } \\
\text { Proportion }\end{array}$ & $\begin{array}{l}\text { Specificity } \\
\text { True- } \\
\text { Negative } \\
\text { Proportion }\end{array}$ & $\begin{array}{c}\text { False- } \\
\text { Positive } \\
\text { Proportion } \\
\text { (1-Specificity) }\end{array}$ \\
\hline 63 & $\begin{array}{l}\text { Janosz et al. } \\
(2008)\end{array}$ & $8-12$ & $\begin{array}{l}\text { Growth Mixture Modeling of unstable } \\
\text { engagement pathways }\end{array}$ & 13300 & 0.030 & 0.266 & 0.787 & 0.932 & 0.068 \\
\hline 64 & $\begin{array}{l}\text { Kupersmidt and } \\
\text { Coie (1990) }\end{array}$ & 5 & Rejection & 99 & 0.182 & 0.313 & 0.278 & 0.864 & 0.136 \\
\hline 65 & & 5 & Aggression & 99 & 0.182 & 0.538 & 0.389 & 0.926 & 0.074 \\
\hline 66 & & 5 & Absences & 99 & 0.182 & 0.368 & 0.389 & 0.852 & 0.148 \\
\hline 67 & & 5 & Failing grades & 99 & 0.182 & 0.235 & 0.222 & 0.840 & 0.160 \\
\hline 68 & $\begin{array}{l}\text { Lee and Staff } \\
(2007)\end{array}$ & $9-10$ & Working over 20 hours per week & 4985 & 0.058 & 0.090 & 0.424 & 0.736 & 0.264 \\
\hline 69 & $\begin{array}{l}\text { Mahoney and } \\
\text { Cairns (1997) }\end{array}$ & $7-12$ & $\begin{array}{l}\text { Middle School Extracurricular Activity } \\
\text { (Students not involved in more than } 1 \text { activity) }\end{array}$ & 378 & 0.151 & 0.171 & 0.947 & 0.184 & 0.816 \\
\hline 70 & & $7-12$ & $\begin{array}{l}\text { High School Extracurricular Activity (Students } \\
\text { not involved in more than } 1 \text { activity) }\end{array}$ & 337 & 0.095 & 0.168 & 0.969 & 0.498 & 0.502 \\
\hline 71 & & $7-12$ & $\begin{array}{l}\text { Middle school at risk category (aggressive, } \\
\text { unpopular, low achievement) }\end{array}$ & 378 & 0.151 & 0.387 & 0.632 & 0.822 & 0.178 \\
\hline 72 & & $7-12$ & $\begin{array}{l}\text { High school at risk category (aggressive, } \\
\text { unpopular, low achievement) }\end{array}$ & 337 & 0.095 & 0.229 & 0.500 & 0.823 & 0.177 \\
\hline 73 & Mahoney (2000) & $4-12$ & No extracurricular activity participation & 653 & 0.156 & 0.416 & 0.559 & 0.855 & 0.145 \\
\hline 74 & & $4-12$ & $\begin{array}{l}\text { Cluster analysis of low academics, popularity, } \\
\text { SES and high aggression }\end{array}$ & 653 & 0.156 & 0.543 & 0.490 & 0.924 & 0.076 \\
\hline 75 & McCaul (1989) & $10-12$ & Grades (Averaged C\&D's and below) & 2635 & 0.223 & 0.411 & 0.368 & 0.849 & 0.151 \\
\hline 76 & & $10-12$ & Test Score Quartiles (Lowest Quartile) & 2635 & 0.223 & 0.384 & 0.492 & 0.774 & 0.226 \\
\hline 77 & & $10-12$ & SES Quartiles (Lowest Quartile) & 2635 & 0.223 & 0.336 & 0.475 & 0.731 & 0.269 \\
\hline 78 & McNeal (1997) & $10-12$ & Whether or Not Students Worked & 20493 & 0.082 & 0.082 & 0.591 & 0.409 & 0.591 \\
\hline 79 & & $10-12$ & $\begin{array}{l}\text { Employed in retail, service, manufacturing or } \\
\text { other }\end{array}$ & 20493 & 0.082 & 0.110 & 0.415 & 0.699 & 0.301 \\
\hline 80 & $\begin{array}{l}\text { Mensch and } \\
\text { Kandel (1988) }\end{array}$ & $9-12$ & Smoking one or more packs per day & 11661 & 0.223 & 0.337 & 0.539 & 0.695 & 0.305 \\
\hline 81 & & $9-12$ & Used marijuana 100 or more times ever & 11661 & 0.223 & 0.346 & 0.275 & 0.850 & 0.150 \\
\hline 82 & & $9-12$ & Ever used cocaine & 11661 & 0.223 & 0.280 & 0.230 & 0.830 & 0.170 \\
\hline 83 & & $9-12$ & $\begin{array}{l}\text { Used other illicit drugs } 40 \text { or more times } \\
\text { (excluding marijuana) }\end{array}$ & 11661 & 0.223 & 0.356 & 0.163 & 0.915 & 0.085 \\
\hline 84 & & $9-12$ & Women who became pregnant before age 19 & 5763 & 0.251 & 0.526 & 0.620 & 0.813 & 0.187 \\
\hline 85 & Muthén (2004) & $7-12$ & $\begin{array}{l}\text { Growth in mathematics test scores using } \\
\text { growth mixture modeling }\end{array}$ & 2757 & 0.147 & 0.693 & 0.896 & 0.932 & 0.068 \\
\hline 86 & $\begin{array}{l}\text { Pagani et al. } \\
\text { (2008) }\end{array}$ & K-6 & $\begin{array}{l}\text { Three risk factors (mother less than a H.S. } \\
\text { diploma, single parent family, retained) }\end{array}$ & 1605 & 0.303 & 0.971 & 0.068 & 0.999 & 0.001 \\
\hline 87 & Roderick (1994) & $4-12$ & Retained at least once between K-8 & 707 & 0.465 & 0.798 & 0.626 & 0.862 & 0.138 \\
\hline 88 & & $4-12$ & Retained at least twice between K-8 & 707 & 0.465 & 0.938 & 0.319 & 0.981 & 0.019 \\
\hline 89 & $\begin{array}{l}\text { Sandefur et al. } \\
\text { (1992) }\end{array}$ & $8-12$ & $\begin{array}{l}\text { Student's family is neither two parent, step } \\
\text { parent or single parent }\end{array}$ & 5246 & 0.264 & 0.483 & 0.050 & 0.981 & 0.019 \\
\hline 90 & & $8-12$ & $\begin{array}{l}\text { Change from two-parent, single parent or step } \\
\text { parent to neither between ages } 14-17\end{array}$ & 5246 & 0.264 & 0.616 & 0.209 & 0.953 & 0.047 \\
\hline 91 & & $8-12$ & Student's parents are step or single parent & 5246 & 0.264 & 0.332 & 0.279 & 0.798 & 0.202 \\
\hline 92 & & $8-12$ & Not intact two parent family from ages $14-17$ & 5246 & 0.264 & 0.398 & 0.694 & 0.623 & 0.377 \\
\hline 93 & Silver et al. (2008) & $7-12$ & Two or more Fs in Middle School & 48561 & 0.520 & 0.600 & 0.300 & 0.783 & 0.217 \\
\hline 94 & & $9-12$ & $\begin{array}{l}\text { Low standardized test scores (9th grade } \\
\text { below/far below basic) }\end{array}$ & 48561 & 0.520 & 0.540 & 0.727 & 0.329 & 0.671 \\
\hline
\end{tabular}

Bowers, Sprott \& Taff (2013) Do We Know Who Will Dropout? 
Table 2 continued

\begin{tabular}{|c|c|c|c|c|c|c|c|c|c|}
\hline ID & Study & $\begin{array}{l}\text { Grade } \\
\text { Level }\end{array}$ & Dropout Indicator & $\begin{array}{l}\text { Sample } \\
\text { Size }\end{array}$ & $\begin{array}{l}\text { Dropout } \\
\text { Rate }\end{array}$ & $\begin{array}{l}\text { Precision } \\
\text { Positive } \\
\text { Predictive } \\
\text { Value }\end{array}$ & $\begin{array}{l}\text { Sensitivity } \\
\text { True- } \\
\text { Positive } \\
\text { Proportion }\end{array}$ & $\begin{array}{l}\text { Specificity } \\
\text { True- } \\
\text { Negative } \\
\text { Proportion }\end{array}$ & $\begin{array}{c}\text { False- } \\
\text { Positive } \\
\text { Proportion } \\
\text { (1-Specificity) }\end{array}$ \\
\hline 95 & & $9-12$ & Over age for typical 9th grader & 48561 & 0.520 & 0.710 & 0.232 & 0.897 & 0.103 \\
\hline 96 & & $9-12$ & Does not pass algebra grade 9 & 48561 & 0.520 & 0.650 & 0.788 & 0.541 & 0.459 \\
\hline 97 & & $9-12$ & Attends more than one high school & 48561 & 0.520 & 0.680 & 0.235 & 0.880 & 0.120 \\
\hline 98 & $\begin{array}{l}\text { South et al. } \\
(2007)\end{array}$ & $7-12$ & $\begin{array}{l}\text { Mobility (student resided at residence one } \\
\text { year or less) }\end{array}$ & 8516 & 0.033 & 0.059 & 0.047 & 0.975 & 0.025 \\
\hline 99 & $\begin{array}{l}\text { Suh and Suh } \\
\text { (2007) }\end{array}$ & $7-12$ & Low GPA & 6192 & 0.153 & 0.159 & 0.078 & 0.925 & 0.075 \\
\hline 100 & & $7-12$ & Low SES & 6192 & 0.153 & 0.166 & 0.113 & 0.898 & 0.102 \\
\hline 101 & & $7-12$ & Suspended & 6192 & 0.153 & 0.181 & 0.154 & 0.874 & 0.126 \\
\hline 102 & & $7-12$ & Any 1 Flag Only & 6192 & 0.153 & 0.171 & 0.346 & 0.697 & 0.303 \\
\hline 103 & & $7-12$ & Any 2 Flags & 6192 & 0.153 & 0.325 & 0.381 & 0.857 & 0.143 \\
\hline 104 & & $7-12$ & 3 Flags & 6192 & 0.153 & 0.477 & 0.142 & 0.972 & 0.028 \\
\hline 105 & Troob (1985) & $9-12$ & Failed 4 or more credits first term grade 9 & 10142 & 0.261 & 0.852 & 0.469 & 0.971 & 0.029 \\
\hline 106 & & $9-12$ & First term grade 9 low or failing GPA & 9808 & 0.258 & 0.836 & 0.570 & 0.961 & 0.039 \\
\hline 107 & & $9-12$ & 16 or more absences in first term grade 9 & 11068 & 0.257 & 0.831 & 0.467 & 0.967 & 0.033 \\
\hline 108 & $\begin{array}{l}\text { Warren and Lee } \\
(2003)\end{array}$ & $8-12$ & Employed in grade 10 & 14787 & 0.063 & 0.068 & 0.260 & 0.761 & 0.239 \\
\hline 109 & & $8-12$ & Employed 1-20 hours per week & 14787 & 0.063 & 0.041 & 0.110 & 0.826 & 0.174 \\
\hline 110 & & $8-12$ & Employed 21 or more hours per week & 14787 & 0.063 & 0.126 & 0.140 & 0.935 & 0.065 \\
\hline
\end{tabular}

* Indicates studies that predicted dropout rather than measured it.

+ Multiple flags were two to three out of the following risk factors: ever previously dropped out, had a child, attended six or more schools, high absenteeism, being overage for grade, low grades, having a sibling who dropped out, unsure of graduating from high school, spends less than 1 hour a week on homework.

++ High risk group defined as one or more indicators: dependent children, ever married, with disabilities, living with neither parent, lone-parent, parent had less than postsecondary education, parents not working, both parents work blue collar jobs, father's education unknown.

\section{RESULTS}

In this section we present the results of the analysis of 110 dropout indicators across the 36 studies. We first present each of the 110 dropout indicators along with the recalculations of precision, specificity and sensitivity for each flag. We then propose the use of relative operating characteristic (ROC) analysis from the systems detection theory literature as a useful procedure to compare each of the different dropout flags using a measure of accuracy based on each indicator's differences in sensitivity and specificity and provide an initial example using ten flags from one of the included studies. Finally, we present the full ROC analysis of all 110 dropout flags to examine the accuracy of reported dropout indicators to date and determine which dropout flags are the most accurate.

Bowers, Sprott \& Taff (2013) Do We Know Who Will Dropout?

\section{Precision, Sensitivity, Specificity}

Table 2 presents our findings, providing a description of each dropout indicator along with the calculated values for each of the 110 dropout indicators across the 36 studies. We assigned an ID to each indicator and provided the grade level at which the indicator was calculated at for each study's sample, a description of each dropout flag, the sample size, dropout rate for the sample, and the precision, true-positive proportion (the sensitivity or "hits"), truenegative proportion (the specificity), and the false-positive proportion ("false alarms"). From the perspective of flags that indicate a high risk of dropping out, the precision can be interpreted as the percent of students with the flag who dropped out, the true positive-proportion is the percent of all of the dropouts who had the flag, and the false-positive proportion is the percent of the graduates who had the flag.

For the first time in the dropout indicator literature, the results in Table 2 provide a means to examine the variability across dropout 
indicator studies in sample context, flags tested, and indicators of precision, sensitivity and specificity. Overall, the studies varied considerably in the grade level at which each flag was calculated, the dropout flags, the sample size of each study and the dropout rates in each context. Grade level ranged from examining indicators in kindergarten and first grade up through the final semesters of high school in grade 12. Sample sizes ranged from a low of 99 through almost 50,000 students. Dropout rates reported also varied considerably, from a low of $3 \%$ to a high of $57 \%$, depending on the context and the number of grade levels included, as samples that included only students from higher grade levels, such as grade 10 or higher, already had experienced students dropping out such that students at earlier grade levels were not captured in the studies. Of note, the studies of the large urban schools districts, Chicago, Los Angeles, and Philadelphia, all had extremely high dropout rates. This issue of the high variability in not only the dropout rates between each sample, but also the size of the samples, is rarely discussed in the dropout identification literature. We discuss this issue at further length below.

Precision was the most commonly reported metric across the studies, and precision ranged across the reported flags from a low of 0.041 for flag number 109 , student employed 1-20 hours per week (Warren \& Lee, 2003), in that only $4.1 \%$ of the students with the flag dropped out, to a high of 0.971 for flag number 86 , three risk factors of mother less than a high school degree, single parent family and student retained (Pagani et al., 2008), in which 97.1\% of the students with the flag dropped out. However, a focus exclusively on precision is problematic. As a measure of the percentage of students who had the flag who dropped out, while almost all of the students may have a flag and drop out, precision alone does not provide an indication of the proportion of all of the dropouts that are identified by the flag. This is because the precision calculation focuses on a proportion based on the predictor, and as noted in Figure 1, is calculated as a proportion of the row marginal total. Thus, precision gives an incomplete indication of the number of students missed by the flag. In contrast, the sensitivity, or true-positive proportion, provides a means to examine the percent of students who dropped out who had the flag.

As an example of this issue, for the Pagani et al. (2008) flag, number 86 , while $97.1 \%$ of the students with the flag dropped out (precision), only $6.8 \%$ of all of the dropouts had the specific reported combination of flags (sensitivity, or true-positive proportion). Thus, while of interest, in the search for a dropout flag or combination of flags that identifies the majority of the students who will drop out, one must take into account both the precision and the true-positive proportion. The true-positive proportion varied across the studies from a low of 0.044 for flag number 13, student has all four of low attendance, unsatisfactory behavior, failed math and failed English in grade 6 (Balfanz, et al., 2007), to a high of 0.969 for flag 70, student involved in no more than one extracurricular activity in high school (Mahoney \& Cairns, 1997), indicating that $96.9 \%$ of the students who dropped out in the Mahoney and Cairns sample did not participate in more than one extracurricular activity in high school. In addition, the falsepositive proportion is equally of interest, since it captures an indication of the number of graduates misidentified as potential dropouts. As an assessment of a dropout flag, one would want the false-positive proportion, the false alarms, to be low. Across the studies, the false-positive proportion varied from a low of 0.001 for flag 86, three risk factors (Pagani, et al., 2008), to a high of 0.816 for flag 69 , student not involved in more than one extracurricular activity in middle school (Mahoney \& Cairns, 1997), indicating that for students in the Mahoney and Cairns sample, $81.6 \%$ of the graduates had this specific dropout flag, suggesting poor specificity for this flag. Hence, as can be seen from Table 2, examining the true- and false-positive proportions is important since these two proportions can vary substantially from each other. As will be discussed below, this is because each proportion is independent from the other because it is calculated from a different subgroup. Stated a different way, in Figure 1, these two proportions are calculated using different column marginal totals, which means that the true-positive proportion is calculated from the dropouts and the false-positive from the graduates. To date, this point has not been articulated in the dropout prediction literature.

Thus, we found a large amount of variance across each of the studies. This makes interpretation of the findings presented in Table 2 difficult, given the large number of dropout flags as well as the differences between the flags. What is needed is a way to visualize the differences between these calculations that aids in interpreting the accuracy of each dropout indicator.

\section{The Relative Operating Characteristic (ROC)}

One way to visualize these differences is to take the false-positive proportion and the true-positive proportions from Table 2 as an $(\mathrm{x}, \mathrm{y})$ coordinate system. Known in the signal detection theory literature as a Relative Operating Characteristic, or a Receiver Operating Characteristic, an "ROC" calculation (Fawcett, 2004; Hanley \& McNeil, 1982; Swets, 1988; Vivo \& Franco, 2008; Zwieg \& Campbell, 1993), the true-positive proportion is plotted against the false-positive proportion for each indicator. As stated by Gleason and Dynarski (2002), the objective for studies examining the indicators of dropout is to find the most predictive flags that identify the majority of students who will drop out while not misidentifying students who graduate as potential dropouts. This inherently is an issue with the difference between accuracy and precision. A dropout indicator may be highly precise, in that almost all of the students with the flag drop out, yet may not be accurate, in that the flag identifies only a small proportion of all of the dropouts. The dropout indicator literature to date has lacked an effective method for evaluating the accuracy of reported flags.

Plotting each flag in what is known as an "ROC plot" provides a means to evaluate the accuracy of dropout indicators (see Figure 2). As an initial example of the ROC procedure for comparison of dropout flags, we first plotted just ten different dropout flags from Balfanz et al. (2007) in Figure 2. The Balfanz et al. (2007) study is of interest for this initial comparison because it (a) contained many flags, and (b) contained many combinations of flags. Following the recommendations of signal detection theory (Fawcett, 2004; Hanley \& McNeil, 1982; Swets, 1988; Vivo \& Franco, 2008; Zwieg \& Campbell, 1993), the ROC plot in Figure 2 plots the truepositive proportion against the false-positive proportion. A hypothetical dropout indicator that would correctly identify $100 \%$ of the dropouts and zero percent of the graduates would be plotted at the point $(0,1)$ at the top left-most corner of the plot. The dotted forty-five degree line represents a random guess in which the proportion of true-positives and false-positives is equal. An indicator above the line and approaching the top left-most corner is considered more accurate because it maximizes the proportion of true-positives ("hits") while minimizing the proportion of falsepositives ("false alarms"), while an indicator below the line is less accurate. 


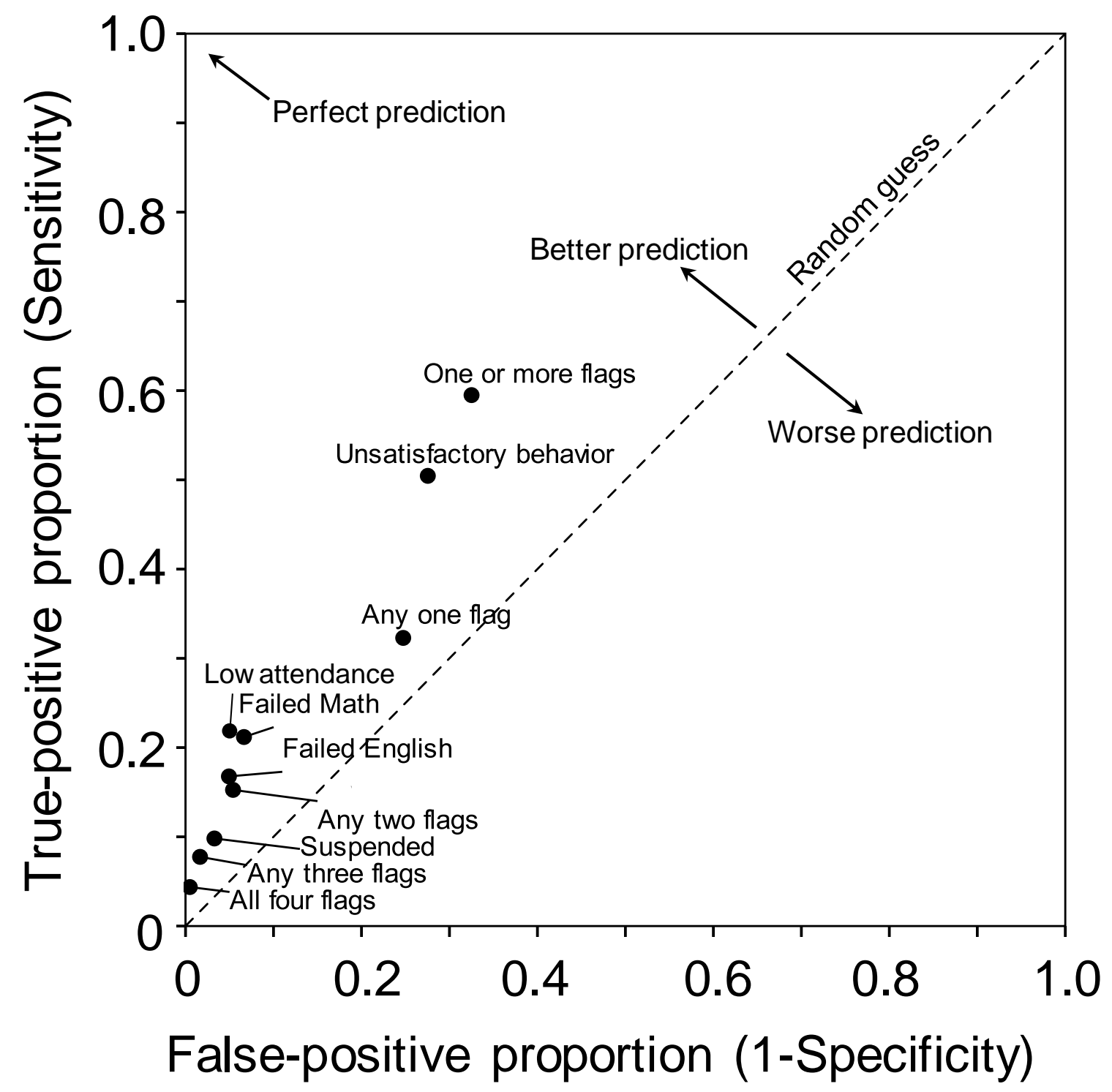

Figure 2: An example of the true-positive proportion plotted against the false-positive proportion for Balfanz et al. (2007) comparing the relative operating characteristics (ROC) of each dropout flag reported in the study. The dotted line represents an equal proportion of true- to false-positive proportions, indicative of a random guess. Below the line indicates a worse prediction, while points closest to the point $(0,1)$ approach perfect classification with $100 \%$ true positives and zero false positives.

As opposed to focusing on just precision or sensitivity as has been the standard practice in the past dropout predictor literature, the ROC plot allows one to visualize each dropout flag within the entire possible ROC space, akin to plotting a bar graph with percentages ranging from 0 as the minimum to $100 \%$ as the maximum. In addition, an ROC analysis is an attractive procedure for evaluating the accuracy of an indicator because it is independent of the prevalence in the sample of the event in question, here dropping out. This is because both the true-positive and false-positive proportions are calculated from different groups (Swets, 1988; Zwieg \& Campbell, 1993), i.e. dropouts and graduates. Thus, the differences in sample sizes and dropout rates described in Table 2 across the different studies are attenuated by some extent by this inherent independence of an ROC analysis from the frequency of the event in the sample.
Figure 2 plots each of the Balfanz et al. (2007) dropout flags in an ROC plot. Here, as reported by Balfanz et al., their best predictor, flag number 9, "one or more flags" from grade six that included low attendance, unsatisfactory behavior, failed math, or failed English, correctly identified about $60 \%$ of the students who dropped out with a true-positive proportion of 0.595 (Table 2 and Figure 2, y-axis). As stated in the original study, these flags correctly identified over half of the dropouts but also missed about $40 \%$ of the students who did eventually drop out. However, less attention was paid in the study to the false-positive proportion of 0.325 (Table 2 and Figure $2 \mathrm{x}$-axis) indicating that $32.5 \%$ of the graduates also had one or more of these four flags. The ROC analysis identifies the "one or more flags" as the more accurate indicator from the study because it is the closest to the upper left corner of the plot. Moreover, the power of the ROC analysis lies in the ability to compare different flags. 


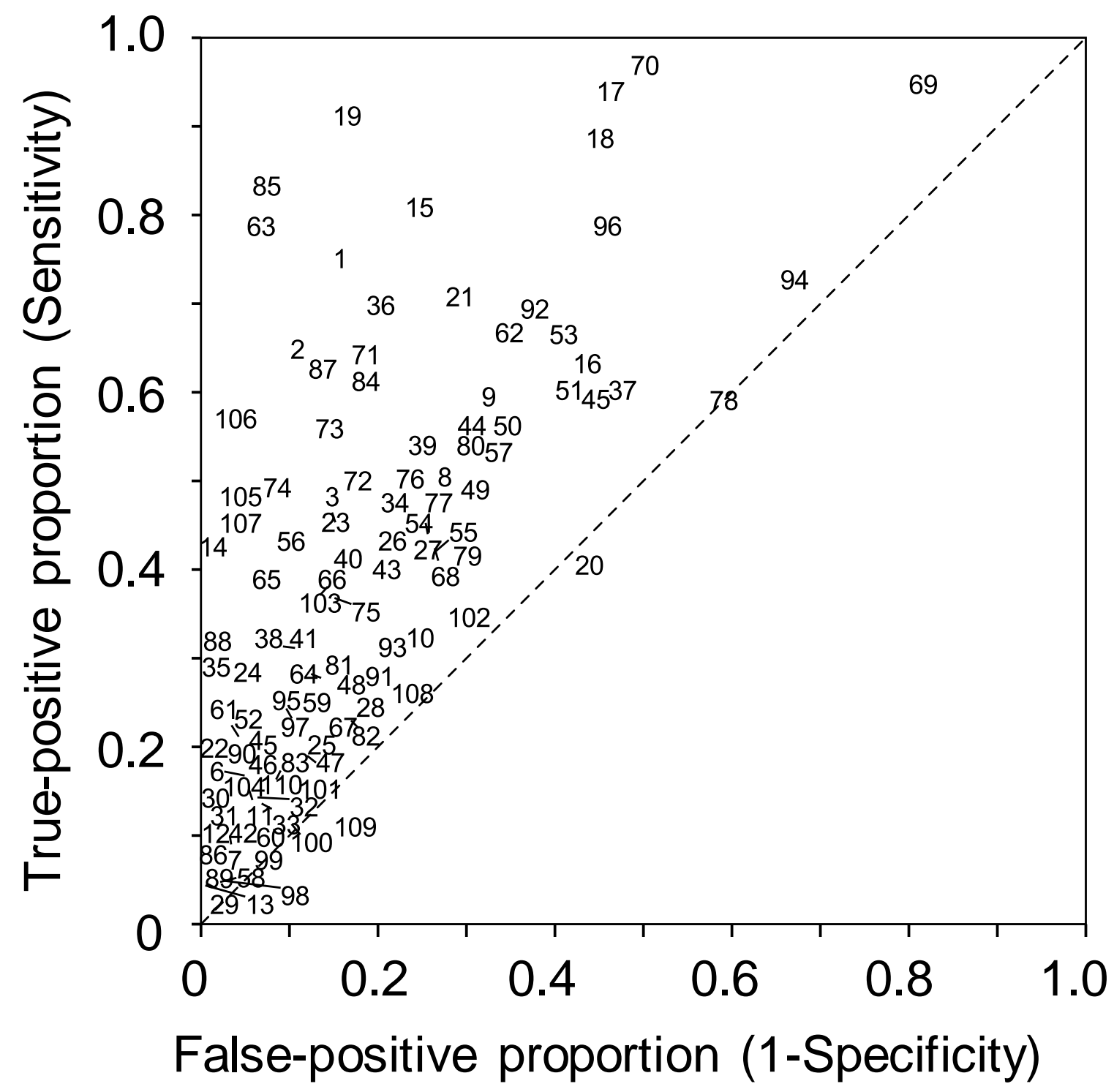

Figure 3: Relative operating characteristics (ROC) of all dropout flags reviewed, plotted as the true-positive proportion against the falsepositive proportion. Each number refers to the dropout indicator ID from Table 2.

As can be seen in Figure 2, for the rest of the flags reported by Balfanz et al., the individual flags decrease in accuracy as they get further from the upper left corner from unsatisfactory behavior, to low attendance, failed math, failed English, suspended. Interestingly, Figure 2 also provides a means to evaluate the practice of combining flags, in that here in the Balfanz et al. study, as flags are combined using the Boolean operator "and" (indicating an intersection) the false-positive proportion decreases with an increase in the precision (see Table 2 and Figure 2). There is a trade-off in that the true-positive proportion also decreased, as is evidenced in the relative decreasing position of each additional flag on the ROC plot, further and further away from point $(0,1)$. However, the use of "or" (indicating an union) of the "one or more flags" indicator appears to be a valuable strategy from the study in helping to increase accuracy, because it is the more accurate predictor by the ROC analysis.

\section{A Comparison of Dropout Indicators Using ROC Analysis}

Figure 3 presents the final full comparative ROC analysis across all 110 dropout flags from the 36 studies. Figure 3 plots the truepositive proportion by the false-positive proportion for each dropout flag numbered according to Table 2. As a point of reference, the "one or more flags" Balfanz et al. (2007) indicator from Figure 2 is labeled in Figure 3 as "9" at point $(0.325,0.595)$. For the first time in the dropout indicator literature, Figure 3 provides a means to examine the accuracy, sensitivity and specificity of each of the reported dropout flags across all of the studies, visualizing each point relative to the rest of the entire ROC space. The purpose of this study is to identify the flags most associated with students who drop out that are (a) accurate, (b) simple to obtain and usable by schools, and (c) under the influence of schools rather than demographics, family SES or neighborhood effects. This analysis provided six main findings. 
First, the use of an ROC plot is an improvement over the past methods of reporting only precision or the true-positive proportions, in that each dropout flag can now be visualized and evaluated for accuracy in the context of the other reported indicators. In examining the Balfanz et al. (2007) flags from Figure 2, now in Figure 3 as points 4 through 13, while flag number 9, "one or more flags", is fairly accurate, it does not appear to be one of the most accurate flags of all of the flags analyzed. The second main finding is that the majority of the dropout flags in Figure 3 cluster near the bottom left of the ROC space. This indicates that while many of these dropout flags had low false-positive proportions, they also had low true-positive proportions, identifying only a small fraction of all of the students who dropped out from each of the samples. The third finding is that many of these dropout indicators are no better than a random guess; with near equal proportions of true-positives and false-positives near the dotted line, such as flag 78 "whether or not a student worked".

Fourth, few studies are near the top of the ROC space in Figure 3 with high true-positive rates. Mahoney and Cairns (1997) provide an interesting example with dropout flags 69 and 70, in which the flag was students involved in no more than one extracurricular activity in middle school or high school, respectively. In Figure 3, flag 69 is near the top right of the ROC space, indicating both a high true- and false-positive proportion. The dropout flag of students in middle school from the Mahoney and Cairns sample who participated in one or no extracurricular activities captures almost all of the students who dropped out, $94.7 \%$ of them; few students drop out who participated in more than one extracurricular activity in middle school. However, this flag also captured $81.6 \%$ of the graduates, in that less than $20 \%$ of the graduates participated in more than one extracurricular activity, indicating that this flag performed poorly, despite its high true-positive proportion. As discussed above, and plotted here, this is an example of the need to consider both the true- and false- positive proportions, which can behave very differently dependent upon the flag under consideration. Conversely, flag 70, students in high school who participated in no more than one extracurricular activity, is much more accurate, with a similarly high true-positive proportion (0.969) but a relatively lower false-positive proportion (0.502). The ROC analysis provides a means to evaluate flags such as these in comparison to all of the others.

The fifth main finding is that overall, the dropout flags 85, 19 and 63 are the most accurate by the ROC analysis, grouping together in the upper left of Figure 3. Interestingly, all three of these studies used a form of multivariate longitudinal analysis, Growth Mixture Modeling (GMM), in which flag 85, Muthén (2004), modeled math achievement trajectories from grades 7-12, flag 19, Bowers and Sprott (2012a), modeled the trajectories of non-cumulative GPA from grades 9-12, and flag 63, Janosz et al. (2008), modeled student engagement trajectories from grades $8-12$. From the ROC analysis, we posit that flag 85 , longitudinal growth in mathematics achievement (Muthén, 2004), is the most accurate of all 110 flags reviewed, in that as a measure of the longitudinal trajectory of student mathematics achievement, it has one of the highest truepositive proportions (highly sensitive) while maintaining a very low false-positive proportion. This finding that the most accurate dropout flags incorporate a form of longitudinal growth modeling supports the dropout-as-a-"life course" literature (Alexander, Entwisle, \& Kabbani, 2001; Bowers, 2010a, 2010b; Jimerson, Egeland, Sroufe, \& Carlson, 2000; Pallas, 2003) that has suggested that the best way to describe the dropout process is not with crosssectional data, but rather as a long-term longitudinal event history that includes a student's trajectory through time in school. Our findings support this literature that has argued for longitudinal analysis, demonstrating that the most accurate indicators of dropout appear to be the longitudinal trajectories of student achievement or engagement in school. Indeed, the point of the GMM method used in each of these three flags is to incorporate a growth trajectory model within a structural equation modeling framework, in which the growth model segment of the GMM models the longitudinal change of students over time, while controlling for demographics (Jung \& Wickrama, 2008; Kaplan, Kim, \& Kim, 2009; Muthén, 2004). In addition, these types of models are mixture models, which sort out the different trajectories from one another, removing and enriching the group of students with the flag to only those students that have statistically similar growth trajectories. This type of GMM analysis is fairly complex and stands in contrast to the majority of the other studies included that used descriptive statistics, cross-tabulations, or logistic regressions to identify a dropout flag. Thus, it appears that for policy and research on dropout flags, growth mixture models that include achievement or engagement trajectories are superior to all of the other flags reviewed.

Nevertheless, for teachers, administrators, schools and districts, while longitudinal analysis is important, one consistent argument from the dropout literature is that these stakeholders need an easyto-calculate flag using data already collected in schools that identifies the majority of the students who drop out and does not incorrectly flag graduates at a high rate. Therefore, our final finding to describe is the next most accurate flags from Figure 3, which includes flag 1, the Chicago on-track indicator including low course credits and failures in grade 9 (Allensworth \& Easton, 2007), flag 15, low non-cumulative GPA (Bowers, 2010b), and flag 2, three or more first semester course failures (Allensworth \& Easton, 2007). As the final main finding, our analysis demonstrates that out of all of the flags reviewed, flag number 1, the Chicago on-track indicator (Allensworth \& Easton, 2007), is the most accurate and most usable dropout indicator. The ROC analysis indicates that in comparison to all but the growth mixture model studies, the on-track indicator is highly accurate, and, as argued by Allensworth and Easton (2007), is usable by schools because it a) includes only data already collected in schools (course credits and failures), b) is easy to calculate by examining if a student is behind on the number of credits to stay on-track to graduate and c) examines if a student has any course failures. In addition, the ontrack indicator provides a means for intervention, in identifying students that need assistance from the school and district to help them obtain the appropriate number of credits to put them on-track for graduation and to perform well enough in the specific courses they have failed to pass. Moreover, these three flags together, flags 1,15 , and 2 , are interesting in that they each include an indication of the performance of the students as measured by low or failing grades. We turn next to a discussion of each of these main findings.

\section{DISCUSSION}

While ROC analysis has been rarely applied to the dropout indicator literature before, it appears to have worked well here as a summary procedure reflecting the accuracy of an indicator relative to the other dropout indicators. Here, we offer the present study as a step towards increasing the ability of researchers and practitioners to compare flags on similar measures through consistent reporting of sensitivity and specificity. However, as presented above, the studies varied remarkably across contexts, 
grade levels, span of data collected, sample sizes, and the types of data that were reported. For future research, we encourage researchers to continue in this line and examine the research using meta-analytic methods, to help control for sample size differences, as well as variation across the contexts. However, given current reporting of dropout indicators as dichotomous, as has been detailed in the signal detection literature (Rice \& Harris, 2005), this is problematic for meta-analysis comparisons because effect size measures are assumed to be normally distributed, as are the standard errors, while any effect size comparison in the dropout literature currently would require point-biserial correlations. Due to this issue, combined with the lack of consistent reporting across studies reported above, we relied here on the use of the ROC comparison method as a first step to improving accuracy reporting across the studies. While outside the scope of the present study, we encourage future research in this domain to examine the use of continuous indicator outcomes, such as ROC "Area Under the Curve" (AUC) analysis (Swets, 1988), which is amenable to effect size comparisons such as meta-analysis (Rice \& Harris, 2005).

Thus, given the present findings for future research we recommend that dropout indicator studies include not only calculations for precision, sensitivity and specificity, but also the raw crosstabulation numbers so that each of these proportions may be recalculated at a later date and included in future ROC studies. As others have called for studies that use inferential statistics to appropriately report effect sizes such that standard meta-analysis techniques can be applied, we stress here that it is important for dropout indicator studies to report these calculations based on the recommendations from signal detection theory. As our review of the studies to date indicated, this literature domain has lacked consistent reporting standards so that claims as to precision and accuracy can be evaluated across studies. In reading the studies nominated for inclusion based on our initial search criteria, we initially thought that there would be many more than 36 studies included in the final analysis. We were dismayed to find that haphazard reporting prevented the inclusion of many studies, since we were unable to recalculate the required numbers, because they were either not reported or were not reported appropriately. This was especially true for many of the dropout indicator studies that are highly cited in this domain, such as Alexander et al. (2001) for example, in which, rather than report the actual sample sizes, n's were reported as ranges. In addition, for the 36 included studies, these had to be read multiple times to find all of the information needed to recalculate the numbers, because different studies provided the information in multiple locations, from methods sections, to tables, to the text, to figures and footnotes. We postulate that some of this is due to the large number of non-peer reviewed reports from research and policy centers, but even many of the peer-reviewed journal articles reported the numbers in an inconsistent format. For future research, we recommend that studies report each of the numbers as an event table as in Figure 1 here, as well as each of the calculations for precision, and true- and false-positive proportions.

One of the main findings here is that the majority of the dropout flags included in this analysis clustered near the lower left of the ROC plot, with low false-positive proportions but also low truepositive proportions. We speculate this is because prior to the use of ROC analysis, this domain has lacked a standard method for determining the accuracy of each of the dropout flags. Rather, it appears that many of the studies focused on precision in the absence of accuracy, driving down the false-positive proportion (increasing specificity) but in turn identifying smaller proportions of all of the students who drop out. This may be a good result. Highly precise and specific dropout flags are useful, even if they are not sensitive. For such flags, almost $100 \%$ of the students with the flag may drop out, and while the flag misses the majority of the dropouts, this information about a specific flag can be informative for schools. Retention, requiring a student to repeat a grade level, as a dropout flag provides a good example of this point. Retention was defined very differently across the studies, ranging from over age in grade 8 or grade 9 , to asking students if they had ever been retained, to examining school records on if the student had ever been retained, to restricting a definition of retention to just specific grade levels, such as middle school. This led to differences in precision and specificity across the studies due to the retention definition, as well as which grade level was included in the definition and how dropout was defined in the study. Nevertheless, for many of the studies that examined retention as a dropout flag, while only a small proportion of all of the dropouts were retained (low sensitivity), the majority of the students retained dropped out (high precision). Thus, as an example here of high precision with low sensitivity, as stated in the extensive literature on the deleterious impact of retaining students (Jimerson et al., 2005; Roderick, 1994), retaining a student is something that schools do to students, and knowing that retaining students is highly predictive of dropout even if not all dropouts are retained, is an important consideration when attempting to decrease dropout rates.

Furthermore, we found that while combining flags using and increases precision, in that students have each of the flags, our results suggest that a better strategy is to combine flags with or, such that students have any one of the nominated flags. We speculate that this focus on the intersection of flags, rather than the union, also contributed to the high amount of clustering of studies on the lower left of the ROC analysis, increasing precision but decreasing sensitivity. It may be that students drop out for many different reasons, and this is supported by the dropout typology literature (Bowers, 2010a; Bowers \& Sprott, 2012b; Fortin, Marcotte, Potvin, Royer, \& Joly, 2006; Janosz, LeBlanc, Boulerice, \& Tremblay, 2000). Thus, different flags may encompass different types of students who are highly likely to drop out, so it stands to reason that multiple non-overlapping flags would cast a wide net and capture the majority of students who drop out. However, as demonstrated in the Balfanz et al. (2007) example in Figure 2, this type of union calculation may experience increased false-positive proportions, especially if each separate flag has a relatively large proportion of false-positives. Thus, we encourage future research to report on both the union of flags as well as the intersection.

Other than the growth mixture models, the results of the ROC analysis indicated that some of the most accurate dropout indicators that use cross-sectional data, focus on low or failing grades. While this is important given that grades are collected regularly in schools for all students and provide an accessible data point with high face validity for teachers and administrators (Allensworth \& Easton, 2007; Bowers, 2010a, 2010b), grades have historically been viewed as a subjective and "hodgepodge" assessment of student ability and academic knowledge, including academic achievement as well as class participation and behavior (Brookhart, 1991; Cizek, Fitzgerald, \& Rachor, 1995-1996; Cross \& Frary, 1999). However, an emerging line of research suggests that teacher-assigned grades are a multi-dimensional assessment of both student academic achievement as well as a student's ability to negotiate the social processes and norms of schooling (Bowers, 2009, 2011; Klapp Lekholm \& Cliffordson, 2008, 2009; 
Willingham, Pollack, \& Lewis, 2002). We posit that low or failing grades may constitute teacher assessment of a student's ability at both the academic components of their courses and social and behavioral components, as represented by their low and failing grades, indicators highly predictive of whether a student will persist in the system. We encourage more work in this area, since our results here suggest that low and failing grades, especially when coupled with a low number of credits in high school, are some of the most accurate indicators of students at risk of dropping out.

\section{CONCLUSION}

In conclusion, our results indicate that while there is high variability across the dropout flags in the literature, there are some indicators that are more accurate than others. The goal of an early warning system, which is the purpose of dropout flags, to warn a school early that a student is at an increased risk of dropping out in the future, is to correctly identify the students who will drop out, without mistakenly flagging students who would have graduated anyway. The costs of poor and inaccurate dropout flags is not only in misspent funds on dropout interventions for students who would have graduated anyway, but also in categorizing students as at-risk when they are not, as well as in missing students who actually are at risk of dropping out. We hope that this study provides a way forward to help future research on dropout identification improve the accuracy of dropout flags, to help identify early which students are the most likely to drop out, and direct the limited funds of schools and districts to the specific needs of each student to help them graduate on time.

\section{RECOMMENDED CITATION FORMAT}

Bowers, A.J., Sprott, R., Taff, S.A. (2013) Do we Know Who Will Drop Out? A Review of the Predictors of Dropping out of High School: Precision, Sensitivity and Specificity. The High School Journal. 96(2), 77-100. doi:10.1353/hsj.2013.0000

\section{REFERENCES}

Alexander, K. L., Entwisle, D. R., \& Kabbani, N. S. (2001). The dropout process in life course perspective: Early risk factors at home and school. The Teachers College Record, 103(5), 760-822.

Allensworth, E. M., \& Easton, J. Q. (2007). What matters for staying on-track and graduating in Chicago public high schools: A close look at course grades, failures, and attendance in the freshman year. Chicago: Consortium on Chicago School Research.

Austin ISD. (1982). "Mother got tired of taking care of my baby." A study of dropouts. Austin, Texas: Austin Independent School District.

Balfanz, R., Bridgeland, J. M., Moore, L. A., \& Hornig Fox, J. (2010). Building a grad nation: Progress and challenge in ending the high school dropout epidemic. Washington D.C.: Civic Enterprises Everyone Graduates Center at Johns Hopkins University America's Promise Alliance.

Balfanz, R., Herzog, L., \& MacIver, D. J. (2007). Preventing student disengagement and keeping students on the graduation path in urban middle-grades schools: Early identification and effective interventions. Educational Psychologist, 42(4), 223-235.
Balfanz, R., \& Legters, N. (2006, July 12). Closing 'dropout factories': The graduation-rate crisis we know, and what can be done about it. Education Week, 25, 42-43.

Berktold, J., \& Carroll, C. D. (1998). Subsequent educational attainment of high school dropouts. Office of Educational Research and Improvement. Retrieved from http://nces.ed.gov/pubsearch/pubsinfo.asp?pubid=98085.

Bowers, A. J. (2009). Reconsidering grades as data for decision making: More than just academic knowledge. Journal of Educational Administration, 47(5), 609-629. doi: $\underline{10.1108 / 09578230910981080}$

Bowers, A. J. (2010a). Analyzing the longitudinal K-12 grading histories of entire cohorts of students: Grades, data driven decision making, dropping out and hierarchical cluster analysis. Practical Assessment Research and Evaluation, 15(7), 1-18. http://pareonline.net/pdf/v15n7.pdf

Bowers, A. J. (2010b). Grades and Graduation: A Longitudinal Risk Perspective to Identify Student Dropouts. Journal of Educational Research, 103(3), 191-207. doi:10.1080/00220670903382970

Bowers, A. J. (2011). What's in a grade? The multidimensional nature of what teacher-assigned grades assess in high school. Educational Research and Evaluation, 17(3), 141-159. doi: $\underline{10.1080 / 13803611.2011 .597112}$

Bowers, A. J., \& Sprott, R. (2012a). Examining the multiple trajectories associated with dropping out of high school: A growth mixture model analysis. Journal of Educational Research, 105(3), 176-195. doi:10.1080/00220671.2011.552075

Bowers, A. J., \& Sprott, R. (2012b). Why tenth graders fail to finish high school: A dropout typology latent class analysis. Journal of Education for Students Placed at Risk, 17(3), 129148. doi:10.1080/10824669.2012.692071

Brookhart, S. M. (1991). Grading practices and validity. Educational Measurement: Issues and Practice, 10(1), 35-36.

Cameron, S. V., \& Heckman, J. J. (1993). The nonequivalence of high school equivalents. Journal of Labor Economics, 11(1), $1-47$.

Cataldi, E. F., Laird, J., \& KewalRamani, A. (2009). High school dropout and completion rates in the United States: 2007. Washington, DC: National Center for Education Statistics, Institute of Education Sciences, U.S. Department of Education.

Cizek, G. J., Fitzgerald, S. M., \& Rachor, R. E. (1995-1996). Teachers' assessment practices: Preparation, isolation and the kitchen sink. Educational Assessment, 3(2), 159-179.

Croninger, R. G., \& Lee, V. E. (2001). Social capital and dropping out of high school: Benefits to at-risk students of teachers' support and guidance. Teachers College Record, 103(4), 548581.

Cross, L. H., \& Frary, R. B. (1999). Hodgepodge grading: Endorsed by students and teachers alike. Applied Measurement in Education, 12(1), 53-72.

Curtis, J., Doss, D., MacDonald, J., \& Davis, W. (1983). Dropout prediction. Paper presented at the Annual meeting of the American Educational Research Association, Montreal.

Dalton, B., Glennie, E., \& Ingles, S. J. (2009). Late high school dropouts: Characteristics, experiences, and changes across cohorts. (NCES 2009-307). Washington, DC: National Center for Education Statistics, Institute of Education Sciences, U.S. Department of Education Retrieved from http://nces.ed.gov/pubs2009/2009307.pdf. 
Doss, D. A. (1986). Ninth grade course enrollment and dropping out. Paper presented at the Annual Meeting of the American Educational Research Association, San Francisco.

Duchesne, S., Vitaro, F., Larose, S., \& Tremblay, R. E. (2008). Trajectories of anxiety during elementary-school years and the prediction of high school noncompletion. Journal of Youth and Adolescence, 2008(37), 1134-1146.

Eide, E. R., \& Showalter, M. H. (2001). The effect of grade retention on educational and labor market outcomes. Economics of Education Review, 20, 563-576.

Ekstrom, R. B., Goertz, M. E., Pollack, J. M., \& Rock, D. A. (1986). Who drops out of high school and why? Findings from a national study. Teachers College Record, 87(3), 356373.

Ensminger, M. E., \& Slusarcick, A. L. (1992). Paths to high school graduation or dropout: A longitudinal study of a first-grade cohort. Sociology of Education, 65(2), 91-113.

Fawcett, T. (2004). ROC Graphs: Notes and practical considerations for researchers. Retrieved from http://citeseerx.ist.psu.edu/viewdoc/download?doi=10.1.1.10. 9777\&rep=rep1\&type=pdf

Finn, J. D., Scott, L. A., \& Fish, R. M. (2008). Educational sequelae of high school misbehavior. Journal of Educational Research, 101(5), 259-274.

Fortin, L., Marcotte, D., Potvin, P., Royer, E., \& Joly, J. (2006). Typology of students at risk of dropping out of school: Description by personal, family and school factors. European Journal of Psychology of Education, 21(4), 363-383.

Frazer, L. (1991). At-risk students three years later: We know which ones will drop out. Paper presented at the Annual Meeting of the American Educational Research Association, Chicago, IL.

French, D. C., \& Conrad, J. (2001). School dropout as predicted by peer rejection and antisocial behavior. Journal of Research on Adolescence, 11(3), 225-244.

Gleason, P., \& Dynarski, M. (2002). Do we know whom to serve? Issues in using risk factors to identify dropouts. Journal of Education for Students Placed at Risk, 7(1), 25-41.

Hanley, J. A., \& McNeil, B. J. (1982). The meaning and use of the area under a receiver operating characteristic (ROC) curve. Radiology, 143, 29-36.

Hess, A. G., \& Lauber, D. (1985). Dropouts from the Chicago public schools: An analysis of the classes of 1982, 1983, 1984. Chicago, IL: Chicago Panel on Public School Finances.

HRSD. (2006). Leaving school - Results from a national survey comparing school leavers and high school graduates 18 to 20 years of age - January 1995. \#1995-00015. Ottawa CA: Human Resources Skills Development Canada.

Janosz, M., Archambault, I., Morizot, J., \& Pagani, L. S. (2008). School engagement trajectories and their differential predictive relations. Journal of Social Issues, 64(1), 21-40.

Janosz, M., LeBlanc, M., Boulerice, B., \& Tremblay, R. E. (2000). Predicting different types of school dropouts: A typological approach with two longitudinal samples. Journal of Educational Psychology, 92(1), 171-190.

Jemal, A., Ward, E., Anderson, R. N., Murray, T., \& Thun, M. J. (2008). Widening of socioeconomic inequalities in U.S. death rates, 1993-2001. PLoS ONE, 3(5).

Jimerson, S. R., Egeland, B., Sroufe, L. A., \& Carlson, B. (2000). A prospective longitudinal study of high school dropouts examining multiple predictors across development. Journal of School Psychology, 38(6), 525-549.

Jimerson, S. R., Pletcher, S. M. W., Graydon, K., Schnurr, B. L., Nickerson, A. B., \& Kundert, D. K. (2005). Beyond grade retention and social promotion: Promoting the social and academic competence of students. Psychology in the schools, 43(1), 85-97.

Jung, T., \& Wickrama, K. A. S. (2008). An introduction to latent class growth analysis and growth mixture modeling. Social and Personality Psychology Compass, 2(1), 302-317.

Kaplan, D., Kim, J.-S., \& Kim, S.-Y. (2009). Multilevel latent variable modeling: Current research and recent developments. In R. E. Millsap \& A. Maydeu-Olivares (Eds.), The SAGE handbook of quantitative methods in psychology (pp. 592612). Thousand Oaks, CA: SAGE Publications.

Kaufman, P. (2004). The national dropout data collection system: History and the search for consistency. In G. Orfield (Ed.), Dropouts in America: Confronting the graduation rate crisis (pp. 107-130). Cambridge, MA: Harvard Education Press.

Klapp Lekholm, A., \& Cliffordson, C. (2008). Discrepancies between school grades and test scores at individual and school level: effects of gender and family background. Educational Research and Evaluation, 14(2), 181-199.

Klapp Lekholm, A., \& Cliffordson, C. (2009). Effects of student characteristics on grades in compulsory school. Educational Research and Evaluation, 15(1), 1-23. doi: 10.1080/13803610802470425

Kronick, R. F., \& Hargis, C. H. (1998). Dropouts: Who drops out and why - and the recommended action (2 ed.). Springfield, IL: Charles C. Thomas.

Kupersmidt, J. B., \& Coie, J. D. (1990). Preadolescent peer status, aggression, and school adjustment as predictors of externalizing problems in adolescence. Child Development, 61, 1350-1362.

Lee, J. C., \& Staff, J. (2007). When work matters: The varying impact of work intensity on high school dropout. Sociology of Education, 80, 158-178.

Mahoney, J. L. (2000). School extracurricular activity participation as a moderator in the development of antisocial patterns. Child Development, 71(2), 502-516.

Mahoney, J. L., \& Cairns, R. B. (1997). Do extracurricular activities protect against early school dropout? Developmental Psychology, 33(2), 241-253.

McCaul, E. (1989). Rural public school dropouts: Findings from high school and beyond. Research in Rural Education, 6(1), 19-24.

McNeal, R. B., Jr. (1997). Are students being pulled out of high school? The effect of adolescent employment on dropping out. Sociology of Education, 70(3), 206-220.

Mensch, B. S., \& Kandel, D. B. (1988). Dropping out of high school and drug involvement. Sociology of Education, 61, 95113.

Moretti, E. (2007). Crime and the costs of criminal justice. In C. R. Belfield \& H. M. Levin (Eds.), The price we pay (pp. 142159). Washington, DC: Brookings Institution Press.

Muenning, P. (2007). Consequences in health status and costs. In C. R. Belfield \& H. M. Levin (Eds.), The price we pay (pp. 125-141). Washington, DC: Brookings Institution Press.

Muthén, B. O. (2004). Latent variable analysis: Growth mixture modeling and related techniques for longitudinal data. In D. Kaplan (Ed.), The Sage handbook of quantitative methodology for the social sciences (pp. 345-370). Thousand Oaks, CA: Sage Publications.

Pagani, L. S., Vitaro, F., Tremblay, R. E., McDuff, P., Japel, C., \& Larose, S. (2008). When predictions fail: The case of unexpected pathways toward high school dropout. Journal of Social Issues, 64(1), 175-194. 
Pallas, A. M. (1989). Conceptual and measurement issues in the study of school dropouts. In K. Namboodiri \& R. G. Corwin (Eds.), Research in the sociology of education and socialization (Vol. 8, pp. 87-116). Greenwich, CT: JAI Press Inc.

Pallas, A. M. (1993). Schooling in the course of human lives: The social context of education and the transition to adulthood in industrial society. Review of Educational Research, 63(4), 409-447.

Pallas, A. M. (2003). Educational transitions, trajectories, and pathways. In J. T. Mortimer \& M. J. Shanahan (Eds.), Handbook of the life course. New York: Kluwer Academic/Plenum Publishers.

Rice, M. E., \& Harris, G. T. (2005). Comparing effect sizes in follow-up studies: ROC area, Cohen's d, and r. Law and Human Behavior, 29(5), 615-620. doi: 10.1007/s10979-0056832-7

Roderick, M. (1994). Grade retention and school dropout: Investigating the association. American Educational Research Journal, 31(4), 729-759.

Rogosa, D. (2005). Statistical misunderstandings of the properties of school scores and school accountability. In J. L. Herman \& E. H. Haertel (Eds.), Uses and misuses of data for educational accountability and improvement: 104th yearbook of the National Society for the Study of Education (pp. 147-174). Malden, Mass.: Blackwell Publishing.

Rouse, C. E. (2007). Consequences for the labor market. In C. R. Belfield \& H. M. Levin (Eds.), The price we pay (pp. 99124). Washington, DC: Brookings Institution Press.

Rumberger, R. W. (1987). High school dropouts: A review of issues and evidence. Review of Educational Research, 57(2), 101-121.

Rumberger, R. W. (2004). Why students drop out of school. In G. Orfield (Ed.), Dropouts in America: Confronting the graduation rate crisis (pp. 131-156). Cambridge, MA: Harvard Education Press.

Sandefur, G. D., Mclanahan, S., \& Wojtkiewicz, R. A. (1992). The effects of parental marital status during adolescence on high school graduation. Social Forces, 71(1), 103-121.

Silver, D., Saunders, M., \& Zarate, E. (2008). What factors predict high school graduation in the Los Angeles Unified School District California Dropout Research Project. Santa Barbara, CA: University of California Santa Barbara.

South, S. J., Haynie, D. L., \& Bose, S. (2007). Student mobility and school dropout. Social Science Research, 36, 68-94.

Suh, S., \& Suh, J. (2007). Risk factors and levels of risk for high school dropouts. Professional School Counseling, 10(3), 297306.

Swanson, C. B. (2004). Sketching a portrait of public high school graduation: Who graduates? Who doesn't? In G. Orfield (Ed.), Dropouts in America: Confronting the graduation rate crisis (pp. 13-40). Cambridge, MA: Harvard Education Press.

Swanson, C. B. (2009). Cities in crisis 2009: Closing the graduation gap: Educational and economic conditions in America's largest cities. Bethesda, MD: Editorial Projects in Education, Inc.

Swets, J. A. (1988). Measuring the accuracy of diagnostic systems. Science, 240(4857), 1285-1293. doi: $10.1126 /$ science. 3287615

Troob, C. (1985). Longitudinal study of students entering high school in 1979: The relationship between first term performance and school completion. New York: New York City Board of Education.
Tyler, J. H. (2003). Economic benefits of the GED: Lessons from recent research. Review of Educational Research, 73(3), 369403.

Vivo, J.-M., \& Franco, M. (2008). How does one assess the accuracy of academic success predictors? ROC analysis applied to university entrance factors. International Journal of Mathematical Education in Science and Technology, 39(3), 325-340. doi: 10.1080/00207390701691566

Waldfogel, J., Garfinkel, I., \& Kelly, B. (2007). Welfare and the costs of public assistance. In C. R. Belfield \& H. M. Levin (Eds.), The price we pay (pp. 160-174). Washington, DC: Brookings Institution Press.

Warren, J. R., \& Lee, J. C. (2003). The impact of adolescent employment on high school dropout: Differences by individual and labor-market characteristics. Social Science Research, 32, 98-128.

Willingham, W. W., Pollack, J. M., \& Lewis, C. (2002). Grades and test scores: Accounting for observed differences. Journal of Educational Measurement, 39(1), 1-37.

Zwieg, M. H., \& Campbell, G. (1993). Receiver-operating characteristic (ROC) plots: A fundamental evaluation tool in clinical medicine. Clinical Chemistry, 39(4), 561-577. 